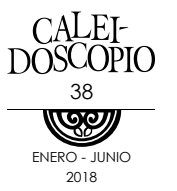

\title{
Cruce de caminos: trabajo informal y economía social
}

Crossroads: informal work and social economy

OCTAVIO MARTÍN MAZA DÍAZ CORTÉS'

ÁNGEL ALEJANDRO POCOROBA GARCÍA²

\section{RESUMEN}

La presente investigación es una discusión teórica que problematiza al autoempleo desde el Trabajo Informal (TI) y la Economía Social y Solidaria (ESS), contextualizándola en la industria textil de Moroleón y Uriangato, Guanajuato. Se centra el autoempleo en las casas-taller porque es en este espacio donde se conjuga una doble cara: la solidaridad y la explotación. A pesar de que informalidad y economía social abordan el tema del autoempleo, éstas corresponden a lógicas diferentes. Ambas son opuestas a la economía capitalista, es decir, se les considera como alternativas al capital, pero se diferencian porque la primera persigue una cultura capitalista y la segunda se basa en prácticas de solidaridad donde el trabajo se antepone al capital. Sin embargo, TI y ESS proporcionan ingresos para aquellas personas que se encuentran en situaciones desfavorables o han sido desplazadas por la economía de mercado.

Palabras clave: trabajo informal, economía social y solidaria, autoempleo, casas-taller.

1 Universidad Autónoma de Aguascalientes

2 Universidad Autónoma de Aguascalientes

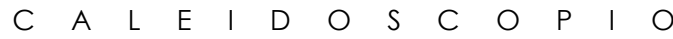


This paper presents a theoretical discussion that problematizes self-employment as Informal Work (IW) and as Social and Solidarity Economy (SSE), as it unfolds in the textile industry of Moroleón and Uriangato, Guanajuato. Self-employment focuses on home-based workshops because it is a double-face space: solidarity on one hand and exploitation on the other. Although informality and social economy address the issue of self-employment, they pertain to different logics. Both are opposite to capitalist economy; in other words, they are considered as alternatives to capital, but they differ because the former pursues a capitalist culture and the latter is based on practices of solidarity where labor is placed before capital. However, IW and SSE provide income for those who are in unfavorable situations or have been displaced by the market economy.

Keywords: informal work, social and solidarity economy, self-employment, home-based workshops.

Desde la década de 1980, la Economía Social y Solidaria (ESS) y el Trabajo Informal (TI) son respuestas a la exclusión económica y social; son consideradas otras formas de economía porque se han destacado por ser alternativas al capital, con distintas racionalidades y lógicas. Además, cuando proponen un desarrollo de lo local como un conjunto de prácticas, reafirman la capacidad de hacer economía (Rodríguez, 2007) frente a la segregación que genera el mercado neoliberal.

El origen de la ESS se explica por la ineficiente capacidad del Estado para generar empleos dentro de la economía de mercado. Plantea un nuevo rol de la economía más articulado con la sociedad: prácticas solidarias que buscan resolver necesidades y sustentos Cotidianos. Para la definición de Economía Informal (EI), Tokman (1992: 23) señala que "los trabajadores (rurales y urbanos) que no gozan de un salario constante y suficiente, así como todos los trabajos a cuenta propia forman parte de la economía informal".

La EI y la ESS cuentan con vínculos estrechos, no sólo por el abanico de posibilidades que abarca sus definiciones conceptuales, sino porque ambas economías operan a través de las relaciones sociales de los agentes. En este sentido, y como lo ha observado la Organización Internacional del Trabajo (OIT, 2014: 51): "cuando los operadores o 
trabajadores informales se encuentran con impedimentos importantes para unirse a las organizaciones de empleadores o los sindicatos existentes o para crear las suyas propias, la estructura organizativa de afiliación más eficaz puede ser el cooperativismo".

No obstante, la EI así como la ESS son opuestas a la economía capitalista, es decir, se les considera como alternativas al capital, pero se diferencian porque la primera (EI) persigue una cultura capitalista; mientras que la segunda (ESS) se basa en prácticas de solidaridad donde el trabajo se antepone al capital. Ambas economías (informal/ social y solidaria) son acciones de los sujetos que buscan alternativas para lograr su sobrevivencia (Véras de Oliviera, 2011; Maza y Ortiz, 2015; Maza y Santoyo, 2016).

La presente investigación discute las perspectivas de la EI y la ESS, centrando el análisis en el autoempleo de las casas-taller, en la industria textil y confección de la zona metropolitana de Moroleón y Uriangato, Guanajuato, México. Se hace uso de los datos obtenidos por el equipo de trabajo al que nos adscribimos: C.A. Trabajo y Relaciones Sociales, de la Universidad Autónoma de Aguascalientes. El estudio se centró en estudiar las relaciones y modelo de producción de las comunidades atípicas de Moroleón y Uriangato, donde el modelo reduce la brecha entre ricos y pobres, ofrece empleo y permite alternativas distintas a las del capitalismo. Ambas propuestas mencionan al autoempleo, pero lo abordan desde lógicas y discusiones distintas (véase Figura 1). 


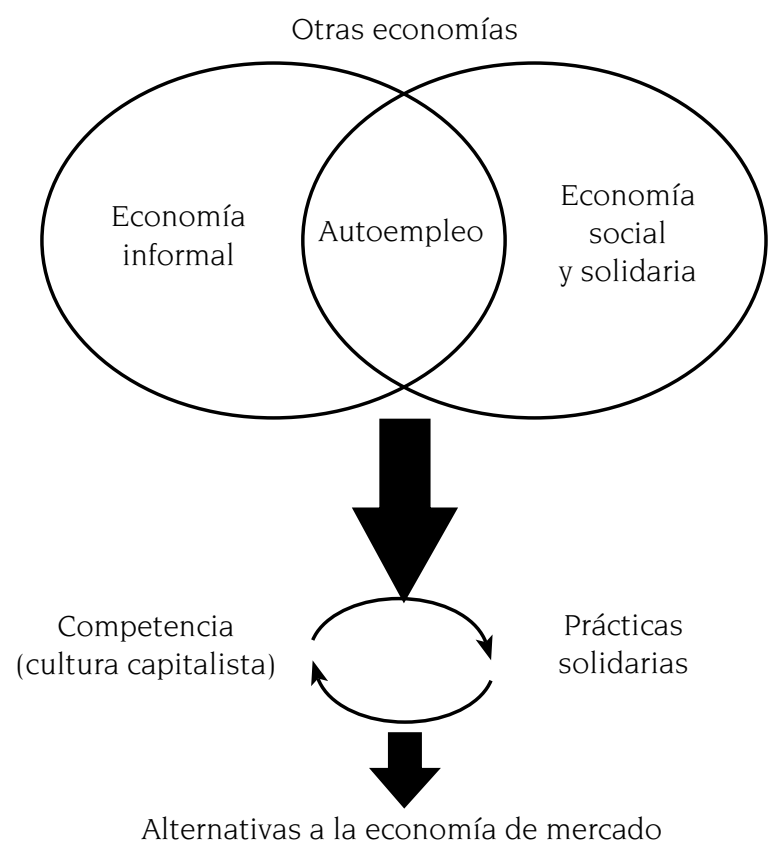

Figura 1. Economía de mercado y sus alternativas

\section{DISCUSIÓN DE LA ECONOMÍA INFORMAL}

En el trabajo de la Organización Internacional del Trabajo (OIT) sobre Kenya en 1972, se hizo presente el término de informalidad y se relacionó con las actividades que llevaban a cabo los trabajadores pobres, las cuales se encontraban ligadas a ocupaciones independientes, de baja productividad y escasa remuneración (Tokman, 1995).

Más tarde, en 1976, El Programa Regional del Empleo para América Latina y el Caribe (PREALC) de la OIT, define al sector informal como empresas pequeñas no modernas y los trabajadores independientes, con exclusión de los profesionales universitarios y trabajos de servicio doméstico. Esta propuesta define a los que ingresaban a este sector como un segmento de población excluido.

Para De Soto et al. (1986), el concepto de informalidad es el resultado de la dificultad que numerosos agentes económicos tienen para cumplir con regulaciones legales que el sistema de mercado exige por 
el sistema de mercado. Es decir, el gobierno estimula la informalidad imponiendo regulaciones excesivas e inapropiadas, de esta manera, las políticas implementadas limitan a las microempresas. Mientras que para Tokman (2011) la informalidad es el resultado del excedente de oferta de mano de obra y la poca o nula respuesta por generar empleo de calidad.

Ante la necesidad de sobrevivir, la gente se ve obligada a buscar alternativas laborales de baja productividad y con bajos ingresos, ya sea produciendo o vendiendo algo. Esta definición cuenta con dos características fundamentales: una de corte legal (similar a la propuesta por De Soto et al. 1986) y la informalidad como alternativa al capital.

Por un lado, el no cumplimiento de leyes es una de las características de la informalidad, pero no es necesariamente su causa. La principal columna del sector informal es la incapacidad real de cumplir con los reglamentos (aun si tuviera la intención de hacerlo), porque el escaso excedente que genera resulta insuficiente para absorber el costo de la legalidad completa (Tokman, 1995). Además, la proliferación de reglamentos y trámites, que se transforman en un número abundante de leyes, provoca el encarecimiento de actividades productivas; para poder ser parte de la formalidad es necesario destinar recursos para acatarlas (tiempo, dinero y esfuerzo). La informalidad no desaparecerá con simples cambios en la ley, ni con la inclusión de informales a la formalidad y, si así fuera, sería solamente en el terreno de lo teórico, pero carecería de fundamentos empíricos.

Por otro lado, los actores sociales al tomar conciencia de su situación y ante el enfrentamiento de la economía de mercado, buscan alternativas al capital para recurrir a una fuente de ingreso, es decir, las personas con sus propias manos buscan generar el capital económico que les fue negado. El sector informal, por lo tanto, puede entenderse como un emprendimiento que existe a causa de los sectores populares que buscan resolver su necesidad de sobrevivir, dentro de un modo de producción excluyente (Maza y Santoyo, 2016).

\section{LÓGICA DE LA ECONOMÍA SOCIAL Y SOLIDARIA}

La Economía Social y Solidaria (ESS) se orienta a la resolución de problemáticas urgentes, en términos de satisfacción de la reproducción de necesidades de la vida de amplios sectores de la población, 
que ante la crisis estructural del mercado de trabajo recurren a la modalidad asociativa. Las diferentes conceptualizaciones remiten principalmente al componente de solidaridad y reciprocidad de los sujetos, quienes protagonizan las experiencias como forma de sostenimiento de esta alternativa económica a la rigidez de los condicionamientos del mercado (Forni y Dzembtowski, 2010).

Algunas perspectivas de la ESS, en particular en América Latina, sostienen que la economía popular sirve como soporte para construir un tipo de economía en donde aparezcan sentidos de cooperación, asociación y solidaridad. En este sentido, Razeto (1999) plantea la necesidad de introducir la solidaridad en la economía misma y que actúe en las diversas fases del ciclo económico: producción, circulación, consumo y acumulación. Ello implica adherir a dicha cadena los preceptos de la solidaridad para lograr actividades que garanticen la sobrevivencia y que, a su vez, resuelvan las necesidades de la vida cotidiana (Razeto, 1999).

Para Coraggio (2011), la ESS busca: a) fortalecer los comportamientos solidarios, y b) generar una economía incluyente pero que no excluya al Estado ni al mercado. Señala que la economía social hace referencia a una situación de las clases que se encuentran vulnerables al desarrollo económico, frente a un proceso de regresión con altísimas tasas de desempleo, subempleo y precarización laboral.

Para ambos autores, la economía social busca el camino a una sociedad más igualitaria y está centrada en el trabajo y no en el capital, ya que ofrece algunos principios-guía que pueden orientar las prácticas políticas de transformación dentro de una matriz de racionalidad reproductiva de la vida de todos (Coraggio, 2011), aspirando a desarrollar nuevas relaciones de consumo sustentadas en la solidaridad, eficaces formas para combatir a las enormes y crecientes injusticias y desigualdades sociales que genera el sistema económico predominante, las cuales se traducen en procesos de desintegración de la convivencia social.

El concepto de ESS se presenta como una alternativa posible a aquellas personas excluidas del mercado formal, donde la economía social configura un aporte y una respuesta diferente al de las organizaciones de la economía de mercado.

Finalmente, partimos de la premisa que la ESS y la EI son alternativas de inclusión de los excluidos, porque implica el uso de la innovación de los recursos para satisfacer necesidades sociales de manera 
sustentable. Al contrastar ambas perspectivas, nos pareció relevante incorporar a la discusión la noción de autoempleo, esto para identificar otras lógicas del mercado, las cuales subyacen del enfoque de competencias relacionado con la economía capitalista.

\section{EL CRUCE: AUTOEMPLEO}

El autoempleo se traduce como un trabajo independiente y no remunerado salarialmente; se trata de trabajadores que auto organizan el desarrollo de su actividad, no están sujetos a órdenes de terceros y son ellos mismos quienes acceden directamente al mercado de bienes y servicios (Valdés, 2005). Además, realizan la actividad de forma habitual o constante, hacen suyos los frutos de su actividad sin que exista una traslación automática de los mismos a un tercero predeterminado (Ariza et al., 2013), y finalmente, no se encuentran sometidos a un contrato de trabajo. El trabajo a cuenta propia es una alternativa para conseguir el ingreso que se les fue negado, pero también es la actividad que escapa ante las regulaciones del Estado.

La descentralización productiva y el empleo de nuevos modos de organización del trabajo están dando como resultado una creciente externalización de las actividades que puede desarrollar la empresa (Valdés, 2005), y consecuentemente el incremento de las actividades de autonomía (por ejemplo, casas-taller), así como el descenso del trabajo dependiente. La figura del trabajo autónomo resulta clave en una coyuntura como la actual, porque se considera como un comportamiento contra-cíclico (Cuadrado et al., 2005).

En este marco, conceptualizamos las actividades realizadas del autoempleo en prácticas alternativas al capital porque supone ciertos indicios de informalidad: la inconsistencia del trabajo, la carencia de protección social: seguridad social, pensión, prestaciones, primas vacacionales, entre otras. Para Cuadrado et al. (2005) y Ariza et al. (2013), el trabajo autónomo es un "empleo refugio" ante los cambios que se están dando en el mercado de trabajo. Sin embargo, para la realidad mexicana, el autoempleo constituye dos lógicas: la sobrevivencia y la cultura capitalista. 
El debate teórico de los enfoques de EI y ESS y de los preceptos del autoempleo lo ubicamos en el estudio de la región productiva de Moroleón y Uriangato, Gto. (zona metropolitana). Los inicios del desarrollo de la industria de Moroleón y Uriangato proceden del siglo XIX, donde se podría apreciar una apropiación y consolidación de las actividades textiles con la elaboración del rebozo alrededor de las décadas de 1940 y 1950. Con esta base productiva, realizada mayormente por mujeres, se abre una coyuntura importante para abrir el mercado nacional y la exportación a los Estados Unidos, escenario en el cual se generó empleo en la "otra economía", donde cientos de hombres y mujeres fueron insertándose en el mercado a partir del aprendizaje del oficio, generando pequeños y medianos talleres -familiares- independientes.

Los datos muestran la fuerte cantidad de empleadores que existen en estas comunidades: en la zona metropolitana cerca de 19\% son trabajadores por cuenta propia y $8.6 \%$ son empleadores. A nivel nacional, la proporción de empleadores es menor (5\%). En Uriangato llama la atención que los patrones son cerca de 10\% de toda la población ocupada, el doble que a nivel nacional. En Moroleón son empleadores 7 de cada cien ocupados (Pérez, 2015).

La descentralización de la industria textil y confección ocasionó que surgiera una reorganización de la mano de obra: el autoempleo, la mano de obra calificada a bajo costo, la inclusión de la producción familiar en la organización, la no delimitación de horario laboral y la falta de una estructura legal y el surgimiento de las casas-taller. En las casas-taller se diseña, confecciona y restauran prendas a familiares, amigos o conocidos, pero también sirven como maquiladoras para grandes empresas, porque son manos de obra a bajo costo (tercerización del empleo).

De acuerdo con Calleja (1984), las casas-taller no se distinguen fácilmente, normalmente se ubican en casas-habitación comunes y no es posible detectar en cuáles se realizan actividades económicas. Además, estos lugares cuentan con un nivel tecnológico inferior a las fábricas, dependen de la mano de obra del operador y sustituyen la necesidad de maquinaria acudiendo a otros talleres de maquila. Al mismo tiempo, las relaciones sociales juegan un papel esencial en las casas-taller, porque es fundamental para el aprendizaje del oficio y, por ende, para que el negocio se mantenga competitivo. 
Asimismo, las investigaciones realizadas en la zona metropolitana de Moroleón y Uriangato (Maza y Ortiz, 2015) han permitido reconocer que las relaciones sociales entre los diferentes actores fluyen y se construyen a través de vínculos de confianza, así como de aspectos como la cooperación y la competencia que existe para poder realizar las actividades de la maquila. En los municipios, la informalidad "está matizada por los fuertes vínculos sociales que existen entre patrones y trabajadores, además de que la capacidad de negociar entre dichos actores se conforma de elementos distintos, tales como la situación del mercado, las calificaciones de los trabajadores y la cercanía que tienen con los patrones" (Maza, 2011).

\section{CONCLUSIONES}

En la mayoría de las casas-taller se hacen presentes las relaciones sociales y el capital social como estrategias competitivas y de sobrevivencia, a partir de ellas es como se dan a conocer las actividades de producción en las casas habitación. La incorporación laboral es directa y a través de lazos familiares o de amistad; además, los vínculos solidarios se hacen presentes durante los periodos de discontinuidad.

De acuerdo a los objetivos planteados en el presente artículo, el autoempleo se centra en dos discusiones teóricas: la EI y la ESS. Primero, la problematización parte de que existen dos economías no convencionales a las cuales se les catalogó como "otra economía", por ser una alternativa al capital, donde el autoempleo es la principal actividad de incorporación. Sin embargo, la economía informal y la economía social (las otras economías) abordan el tema desde distintas perspectivas, aunque ambas mantienen convergencia en tanto son disyuntivas ante el capitalismo.

La ESS aborda el autoempleo desde prácticas solidarias, buscando una sociedad más igualitaria y equitativa. Los actores que se encuentran inmersos en este enfoque anteponen el trabajo sobre el capital. Las personas producen u ofrecen sus servicios a partir de la afiliación a las organizaciones de ESS, creando redes solidarias, donde la ganancia es retroactiva y el precio se establece por las horas de trabajo invertidas en la elaboración del producto, similar a las cooperativas asociativas. No obstante, la ESS se torna prescriptiva porque se dan por naturales las relaciones sociales, de esta manera, se puede abusar 
o aprovechar de los huecos legales para disciplinar y precarizar el trabajo; es decir, el instrumento perfecto para burlar las leyes laborales (Múnera y Nanteuil, 2008).

Por otro lado, el autoempleo desde la informalidad ha sido relacionado con la precariedad y la pobreza, aunque cabe destacar que dentro de este sector conviven dos lógicas: la sobrevivencia y la cultura capitalista. La sobrevivencia no busca tomar riesgos porque la idea es sobrevivir a través del apoyo y la confianza de los actores, creando prácticas solidarias y para que el negocio se mantenga competitivo. Para la cultura capitalista, la informalidad representa una ventaja competitiva, debido a la ausencia de leyes y regulaciones laborales. Ambas lógicas conviven en los mismos espacios pero no deben ser estudiadas de igual manera. La informalidad es un concepto empírico que debe ser estudiado de abajo hacia arriba (Salas, 1992), tomando en cuenta la subjetividad y la cultura como mediadores de las actividades.

Por su parte, el autoempleo reorganiza el modelo económico, ya sea desde la EI o la ESS; incluyendo a los expulsados del mercado, a los que buscan valores solidarios dentro de la economía e inclusive para aquellos que persiguen un enriquecimiento. Hay que entender a las otras economías como economías críticas de la sociedad, donde los estados han fallado para la generación y regulación del empleo; llevando a los sujetos a buscar alternativas al capital, ya sea para sobrevivir, crear redes solidarias o, inclusive, para enriquecerse invirtiendo en los sectores precarios. Replantear la economía implica que exista un mayor diálogo, considerando el análisis sociológico en los fenómenos económicos.

Finalmente, la aproximación teórica y empírica promete próximas vetas por explorar, así como un desarrollo más profundo respecto a diferentes temas: el análisis del capital social de las personas, la educación en la informalidad, el empoderamiento que otorga a las mujeres la economía social y más. Es necesario abordar el tema de la economía no convencional desde diferentes disciplinas y enfoques teóricos y metodológicos, permitiendo mayor aproximación a la realidad social. 
Ariza, J. A., Carbonero, M., Gutiérrez, B. y López, M. C. (2013). El trabajo autónomo: una vía para el mantenimiento del empleo en una sociedad en transformación. CIRIEC-ESPAÑA. Revista de Economía Pública, Social y Cooperativa, 78, 149-174. Recuperado de: http://www. redalyc.org/pdf/174/17429865009.pdf.

Calleja, M. (1984). Dependencia y crecimiento industrial: las unidades domésticas y la producción de calzado en León, Guanajuato. Relaciones Estudios de Historia y Sociedad, 17, 54-85. Recuperado de: http://www.colmich.edu.mx/relaciones25/files/revistas/017/Marga ritaCalleja.pdf.

Coraggio, J. L. (2011). Economía Social y Solidaria: El trabajo antes que el capital. Quito: Universidad Politécnica Salesiana; FLACSO. Recuperado de: http://www.coraggioeconomia.org/jlc/archivos\%20para\%20descargar/economiasocial.pdf.

Cuadrado, J. R., Iglesias, C. y Llorente, R. (2005). El empleo autónomo en España: factores determinantes de su reciente evolución. CIRIEC-ESPAÑA. Revista de Economía Pública, Social y Cooperativa, 52, 175-200. Recuperado de: http://www.redalyc.org/pdf/174/17405210. pdf.

De Soto, H., Ghersi, E. y Ghibellini, M. (1986). El otro sendero: La Revolución Informal. Lima: Editorial El Barranco.

Forni, F. y Dzembtowski, N. (2010). La economía social en Europa y en América Latina. En C. Cross y M. Berger (Eds.), La producción del trabajo asociativo: condiciones, experiencias y prácticas en la economía social, (pp. 337-353). Buenos Aires: Ed. CICCUS.

Maza, O. (2011). La industria textil y el comercio en Uriangato, Gto. Retos y potencialidades productivo-culturales vinculados a una economía global. (Informe de investigación). Aguascalientes: Concyteg/Fomix, Universidad Autónoma de Aguascalientes

Maza, O. y Ortiz, D. (Eds.) (2015). En el mismo barco. Reflexiones sobre la producción el trabajo y las redes sociales: El caso de Uriangato, Guanajuato. Aguascalientes: Universidad Autónoma de Aguascalientes.

Maza, O. y Santoyo, L. (2016). El mercado de trabajo mexicano, las dificultades de la informalidad. Investigación y Ciencia, 24(69), 81-88. Recuperado de: http://www.redalyc.org/pdf/674/67449381011.pdf.

Múnera, L. y Nanteuil, M. (2008). Economie solidaire et critique sociale regards croises Europe-Amérique Latine. Comunicación presentada

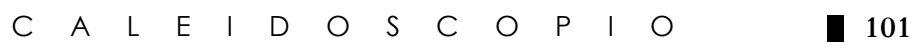


en la $8 e$ conférence internationale de l'I.S.T.R. y $2 e$ conférence internationale $d u$ Réseau E.M.E.S. Barcelona.

OIT (1972). Employment, Incomes and Equality: A Strategy for Increasing Productive Employment in Kenya. Ginebra: OIT. Recuperado de: http:// www.ilo.org/public/libdoc/ilo/1972/72B09_608_engl.pdf.

(2014). La transición de la economía informal a la economía formal.

Ginebra: OIT. Recuperado de: http://www.ilo.org/wcmsp5/groups/ public/@ed_norm/@relconf/documents/meetingdocument/ wcms_218350.pdf.

Pérez, A. (2015). Monografía. En O. Maza y D. Ortiz (Eds.), En el mismo barco. Reflexiones sobre la producción, el trabajo y las redes sociales: El caso de Uriangato, Guanajuato (pp. 15-69). Aguascalientes: Universidad Autónoma de Aguascalientes.

PREALC (1976). El problema del empleo en América Latina: Situación, perspectivas y políticas. Santiago de Chile: OIT.

Razeto, L. (1999). La economía solidaria: concepto, realidad y proyecto. Persona y Sociedad, 13(2). Recuperado de: http://www.luisrazeto. net/content/la-econom\%C3\%ADa-solidaria-concepto-realidad-y-proyecto.

Rodríguez, F. (2007). Economía social, acción pública y política: Hay vida después del neoliberalismo, de José Luis Corraggio. Otra economía, 1(1), 125-127. Recuperado de: http://revistas.unisinos.br/index.php/otraeconomia/article/view/1066.

Salas, C. (1992). ¿Pequeñas Unidades Económicas o Sector Informal? El estado reformado, 45. Recuperado de: http://www.elcotidianoenlinea.com.mx/articulo.asp?id_articulo $=2665$.

Tokman, V. (1992). Beyond regulation. The informal sector in Latin America. Nueva York: Boulder.

(Comp.) (1995). El sector informal en América Latina, dos décadas de análisis. México, D.F.: Consejo Nacional para la Cultura y las Artes.

(2011). Informalidad en América Latina: balance y perspectivas de políticas en Realidad, Datos y Espacio. Revista Internacional de Estadística y Geografía, 2(3), 16-31. Recuperado de: http://www. inegi.org. mx/rde/2011/09/07/informalidad-en-america-latina-balance-y-perspectivas-de-politicas/.

Valdés, A. (2005). La regulación del trabajo por cuenta propia o autónomo: el estado de la cuestión. CIRIEC-ESPAÑA. Revista de Economía Pública, Social y Cooperativa, 52, 149-174. Recuperado de: http://www. redalyc.org/pdf/174/Resumenes/Abstract_17405209_2.pdf. 
Véras de Oliveira, R. (2011). Para discutir os termos da nova informalidade: sobre sua validade enquanto categoria de análise na era da flexibilização. En R. Véras de Oliveira, D. Gomes e I. Targino (Eds.), Marchas e contramarchas da informalidade do trabalho: das origens as novas abordagens (pp. 191-228). Joao Pessoa: Editora da UFPB.

SOBRE LOS AUTORES

Octavio Martín Maza Díaz Cortés (octaviomazadc@gmail.com) es doctor en Estudios Laborales por la Universidad Autónoma MetropolitanaIztapalapa. Profesore investigador titular del Departamento deSociología y Antropología de la Universidad Autónoma de Aguascalientes, sus líneas de investigación se centran en los estudios del trabajo, la informalidad laboral, la precarización del trabajo, las comunidades productivas atípicas y la industria automotriz (ORCID ID: 0000-0002-3991-7751).

Ángel Alejandro Pocoroba García (soc.angelpoc@gmail.com) es licenciado en Sociología por la UAA. Actualmente ocupa el cargo de asistente de investigación del Programa de Política de Drogas del Centro de Investigación y Docencia Económicas A.C. (ORCID ID: 0000-00034132-5023). 
